CONGENITAL HEART DISEASE

\title{
Aortic pressure-area relation in Marfan patients with and without $\beta$ blocking agents: a new non-invasive approach
}

\author{
G J Nollen, B E Westerhof, M Groenink, A Osnabrugge, E E van der Wall, B J M Mulder
}

Heart 2004;90:314-318. doi: 10.1136/hrt.2003.010702

See end of article for authors' affiliations

.....................

Correspondence to:

Dr B J M Mulder,

Department of Cardiology, Room B2-240, Academic Medical Centre

Meibergdreef 9, $1105 \mathrm{AZ}$

Amsterdam, Netherlands;

b.j.mulder@amc.uva.nl

Accepted 11 July 2003
Objective: To investigate the heterogeneous response to $\beta$ blockade in patients with Marfan syndrome by non-invasive assessment of the aortic pressure-area curve.

Design and patients: 25 patients with the Marfan syndrome who used $\beta$ blocking agents (mean (SD) age, 29 (10) years; 20 men, five women), seven without $\beta$ blockade (34 (14) years; five men, two women), and 10 controls (29 (5) years; seven men, three women) underwent magnetic resonance imaging and noninvasive continuous blood pressure measurement. Pressure-area curves were constructed at the level of the descending thoracic aorta. A transition point was defined as the pressure at which the pressure-area relation deviated from its elastic (linear) to the collagen (exponential) course.

Setting: Tertiary referral centre for adult congenital heart disease.

Results: In six patients (five with and one without $\beta$ blockade), a transition point in the pressure-area curve was observed, indicating that the load bearing component was not only elastin but also collagen. In the remaining 26 Marfan patients and in the control subjects a linear pressure-area relation was observed. Conclusions: This new non-invasive method to derive aortic pressure-area curves showed that most patients with Marfan syndrome have a similar pressure-area curve to controls with similar blood pressures. Five patients on $\beta$ blockade showed a transition point in the pressure-area curve which could play a crucial role in the heterogeneous response to $\beta$ blocker treatment in Marfan patients. Patients with a transition at low blood pressures may not benefit from $\beta$ blocking agents.
M arfan syndrome is a heritable disorder of connective tissue resulting in a highly variable degree of premature aortic medial degeneration with a high risk of subsequent dissection or rupture of the aorta. ${ }^{1-3}$ Mortality from aortic dissection and rupture has been related to aortic aneurysm size and expansion rate. ${ }^{45}$ There is evidence that $\beta$ blocking agents reduce the rate of dilatation and the occurrence of dissection in patients with Marfan syndrome; however, some patients respond better than others. ${ }^{6-8}$

A recent study showed that $\beta$ blockade increased aortic distensibility and reduced flow wave velocity only in Marfan patients and not in control subjects. ${ }^{9}$ A heterogeneous response in aortic elasticity after treatment with $\beta$ blockade in Marfan patients has been reported in two other studies. ${ }^{10} 11$ The exact mechanism of this heterogeneity remains undefined. Investigation of the pressure-area relation of the aorta could provide insight into the mechanism behind this heterogeneous response. When, in addition to elastic fibres, collagen fibres are recruited, the pressure-area curve shows a transition from elastic (linear) to a stiff (exponential) course. ${ }^{12} 13$ This transition point is a denominator of aortic elastic behaviour in the normal blood pressure range, and is of additional importance in relation to the forces the aorta can withstand before rupturing. ${ }^{12}{ }^{13}$

Magnetic resonance imaging (MRI) combined with continuous non-invasive blood pressure measurement, such as by the Finapres method, ${ }^{14}$ allows a non-invasive assessment of aortic pressure-area curves. In this study we compared the aortic pressure-area curves of normal subjects with those of patients with Marfan syndrome, with and without $\beta$ blocker treatment. Our aims were first, to investigate whether this non-invasive method is suitable for assessing the aortic pressure-area relation, and second, to gain insight in the heterogeneous response to $\beta$ blockade in patients with Marfan syndrome.

\section{METHODS}

\section{Study population}

We studied 34 consecutive patients with Marfan syndrome, in the age range 19 to 54 years. The diagnosis of Marfan syndrome had been established according to the Ghent criteria. ${ }^{15}$ All subjects underwent MRI immediately followed by finger arterial blood pressure recordings in the supine position with the Finometer. Two patients were excluded because of poor MRI quality. Of the 32 remaining patients, 22 (mean (SD) age, 33 (11) years; 16 men, six women) had previously undergone aortic root replacement. A group of 10 age and sex matched healthy subjects (aged 29 (5) years; seven men, three women) served as a reference population. The characteristics of the study group are shown in table 1.

The study was approved by the local ethics committee, and individual oral and written informed consent was obtained in each patient.

\section{Pressure recording}

Finger arterial blood pressure was recorded with a Finometer, the most recent device in the series of finger arterial blood pressure monitors such as Finapres and Portapres developed by TNO Biomedical Instrumentation (Finapres Medical Systems, Arnhem, Netherlands). The Finometer displays the beat to beat reconstruction of brachial pressure and uses an upper arm cuff for the calibration of this signal. ${ }^{16}{ }^{17}$ Signals are digitised using a sampling rate of $200 \mathrm{~Hz}$ and internally stored. The reconstructed brachial pressure recordings were filtered off-line to derive near-aortic pressures using a generalised filter. ${ }^{18-20}$ Pressure drop because of frictional losses is usually negligible in the pressure transfer over the brachial artery and therefore mean aortic pressure is equal to mean brachial pressure. Pulse shapes, however, are substantially different and this is largely accounted for by filtering. From the reconstructed aortic pressure a beat was selected taking care to match the beat to the heart rate and blood 


\begin{tabular}{|c|c|c|c|}
\hline & $\begin{array}{l}\text { Marfan patients } \\
(n=32)\end{array}$ & $\begin{array}{l}\text { Controls } \\
(n=10)\end{array}$ & p Value \\
\hline \multirow{5}{*}{$\begin{array}{l}\text { Age (years) } \\
\text { Sex (M/F) } \\
\beta \text { Blocker use } \\
\text { Body surface area }\left(\mathrm{m}^{2}\right) \\
\text { Mean blood pressure } \\
(\mathrm{mm} \mathrm{Hg})\end{array}$} & $33(11)$ & $29(5)$ & NS \\
\hline & $25 / 7$ & $7 / 3$ & NS \\
\hline & 25 (78\%) & & \\
\hline & $2.10(0.17)$ & $1.96(0.18)$ & 0.037 \\
\hline & $86(7)$ & $87(8)$ & NS \\
\hline
\end{tabular}

pressure values measured during MRI with a Dinamap oscillometric blood pressure monitor.

\section{MRI measurements}

Imaging was done on a Siemens Magnetom Vision 1.5 Tesla magnetic resonance system (Siemens Medical Systems, Erlangen, Germany). Image acquisition was triggered on the ECG. The entire aorta was imaged in the transverse and oblique sagittal planes using a standard spin echo pulse sequence. Next, a high resolution gradient echo pulse sequence with a velocity encoding gradient (cross sectional fast low angle shot (FLASH): time of repetition (TR) $24 \mathrm{~ms}$, time of echo (TE) $5 \mathrm{~ms}$, flip angle $20^{\circ}$, matrix size $256 \times 256$ ) was applied perpendicular to the aorta. This resulted in multiphase image pairs of modulus and velocity encoded images with a temporal resolution of $24 \mathrm{~ms}$ through the cardiac cycle and a spatial resolution of approximately $1 \mathrm{pixel} / \mathrm{mm}$.

During the flow measurement, the blood pressure and the heart rate were measured using a Dinamap oscillometric blood pressure monitor. A workstation (Sparc Ultra; Sun Microsystems; Mountain View, California, USA) and the FLOW image analysis software (Medis, Leiden, Netherlands) were used for image analysis. Aortic contours were drawn manually on the modulus images of all cardiac phases, and aortic contours were calculated.

\section{Pressure-area curve construction}

For each subject, reconstructed aortic blood pressure and area waveforms were assessed at the descending thoracic aorta at the level of the pulmonary bifurcation (fig l). This level of the pulmonary bifurcation was chosen for the following reasons. First, the filter applied on Finometer recordings produces descending thoracic aortic pressures; second, by choosing this level Marfan patients with an aortic root replacement could also be included.

\section{Calculations}

Transition point ( $\mathrm{TP}, \mathrm{mm} \mathrm{Hg}$ ) was defined as the pressure at which the pressure-area relation deviated from its initial linear behaviour (fig 2A). In this report, E is used for elastance and not for the Young's modulus of the wall material.

Elastance of elastin $\left(\mathrm{E}_{\mathrm{e}}, \mathrm{mm} \mathrm{Hg} / \mathrm{mm}^{2}\right)$ was calculated as the slope of the first (linear) part of the pressure-area curve.

Elastance of collagen $\left(\mathrm{E}_{\mathrm{c}}, \mathrm{mm} \mathrm{Hg} / \mathrm{mm}^{2}\right)$ was calculated as the slope of the exponential part of the pressure-area curve after the transition point.

Distensibility ( $\mathrm{D}, \mathrm{mm} \mathrm{Hg}^{-1}$ ) was calculated by means of the equation:

$$
\mathrm{D}=\frac{\Delta \mathrm{A}}{\mathrm{A}_{\min } \times \Delta \mathrm{P}}
$$

in which $\Delta \mathrm{A}=$ area change $\left(\mathrm{mm}^{2}\right), \mathrm{A}_{\min }=$ minimum area $\left(\mathrm{mm}^{2}\right)$, and $\Delta \mathrm{P}=$ pressure change $(\mathrm{mm} \mathrm{Hg})$.

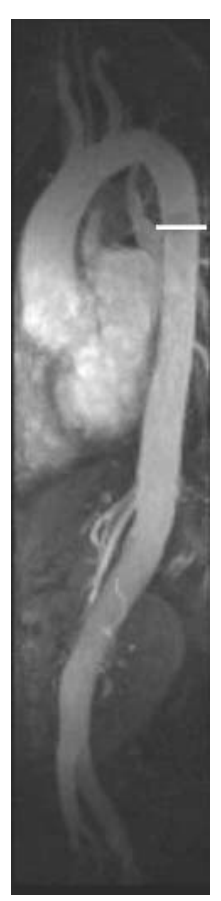

Figure 1 Aortic site at which the pressure-area curve was constructed. The arterial pressure waveform is usually almost identical to the aortic area waveforms. ${ }^{21}$ The area waveforms assessed by magnetic resonance imaging were then matched in time with the reconstructed aortic pressure waveform.

\section{Statistics}

Data are given as mean (SD). Differences between the groups were assessed by the unpaired Student $t$ test. Logistic regression analysis was done to assess independent
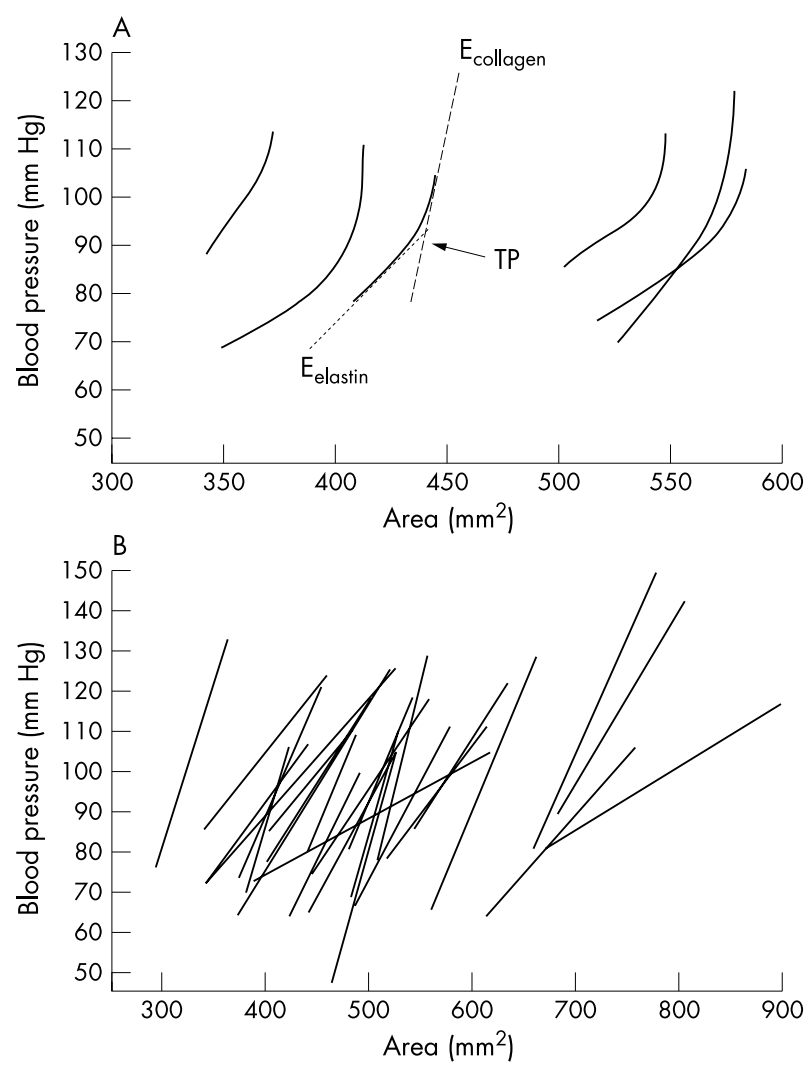

Figure 2 Pressure-area curves (solid lines) of (A) six Marfan patients with a transition point and (B) 26 without a transition point. Dotted line: elastance of elastin $\left(E_{e}\right)$. Striped line: elastance of collagen. $E$, elastance; $T P$, transition point. 
predictors of the occurrence a transition point. Statistical analysis was undertaken using the SPSS statistical package (SPSS Inc, Chicago, Illinois, USA). The level of significance was set at $\mathrm{p}<0.05$.

\section{RESULTS}

\section{Pressure recording}

Reconstructed brachial systolic pressures were close to the Dinamap oscillometric systolic pressures, at 114 (10) $v 115$ (10) $\mathrm{mm} \mathrm{Hg}$, respectively (NS). Reconstructed brachial diastolic pressures differed significantly from the Dinamap oscillometric diastolic pressures (73 (8) and 61 (9) $\mathrm{mm} \mathrm{Hg}$; $\mathrm{p}<0.01)$. Reconstructed aortic heart rate was similar to the heart rate during MRI, at 63 (11) v 64 (11) beats/min (NS).

\section{Pressure-area curves and transition point}

Pressure-area curves were successfully recorded in all 42 subjects. In the group of 10 control subjects no transition point in the pressure-area curves was observed. However, in six of the 32 patients with the Marfan syndrome (19\%), a transition point in the pressure-area curve was demonstrated (table 2). We found no significant difference in the occurrence of a transition point in the pressure-area curve between Marfan patients with $\beta$ blocker treatment and those without (table 2). There was no difference in the slope of the linear part of the pressure-area curve $\left(\mathrm{E}_{\mathrm{e}}\right)$ between control subjects, patients without a transition point, and patients with a transition point (table 3 ). There was a trend towards decreased distensibility in patients with a transition point in the pressure-area curve compared with those without (table 3).

\section{Aortic dimensions and distensibility}

Distensibility was decreased in all Marfan patients compared with the control subjects, at $3.3(1.4) \vee 4.6(0.9) \times 10^{-3}$ $\mathrm{mm} \mathrm{Hg}{ }^{-1}$, respectively $(\mathrm{p}<0.02)$. Descending aortic diameters were increased in the Marfan patients, at 24 (2) $\mathrm{mm} v$ 20 (3) $\mathrm{mm}$ in the controls $(\mathrm{p}<0.01)$. The difference in aortic dimensions or distensibility between Marfan patients with and without $\beta$ blocker treatment was not significant (table 2 ).

\section{DISCUSSION}

In this study we describe a new non-invasive method to evaluate aortic pressure-area relations in individuals with Marfan syndrome, with and without $\beta$ blocker treatment, and in normal subjects. In six Marfan patients (five with and one without $\beta$ blockade) a transition point in the pressurearea curve was observed, indicating that the load bearing component contained collagen as well as elastin. In the resting blood pressure range, we found no significant differences between Marfan patients with and without $\beta$ blocker treatment in aortic distensibility, the slope of the linear part of the pressure-area curve, blood pressure, or aortic dimensions.

\section{Pressure recording}

Aortic blood pressures can only be recorded invasively, and reconstruction techniques are helpful tools to estimate more central pressures from non-invasive recordings. As it is not yet possible to use the Finometer front end in the MRI scanner, pressure recording was done immediately after imaging. Both measurements were assessed under similar circumstances, confirmed by the similarity of heart rate and systolic blood pressure in the two measurements. There was a significant difference between Finometer brachial diastolic pressures and Dinamap oscillometric diastolic pressures. It is well known from published reports that Dinamap oscillometric readings are usually below intra-arterial brachial pressure, especially diastolic pressure..$^{22}$

\section{Pressure-area curves and transition point}

Several studies have reported aortic pressure-area curves with invasive local intravascular measurements, ${ }^{24-26}$ while others used brachial pressure measured by a sphygmomanometer as a surrogate for aortic pressure. ${ }^{27}{ }^{28}$ MRI combined with non-invasive continuous measured blood pressuresuch as by the Finapres method-allows non-invasive assessment of aortic pressure-area curves. ${ }^{14}$ Because the relation between pressure and area is not linear, the pressure-area curve gives additional information on the load bearing component. Over a century ago Roy recognised that the more an artery is stretched the harder it becomes to stretch it further. ${ }^{29}$ This phenomenon occurs because at lower pressures elastin is the major load bearing component. Collagen fibres, which are far stronger but more difficult to stretch, are recruited progressively as the load increases. In the pressure-area curve this process can be seen as a transition from the elastic (linear) course to a stiff (exponential) course. The blood pressure at which this transition occurs is defined as the transition point. Bader studied the pressure-area relation in elderly people and showed that with increasing age, the great arteries became more dilated and the elastin determined (linear) part of the pressure-area relation shifted to lower pressures. ${ }^{13}$ As changes in the aortic tunica media in the Marfan syndrome do not differ essentially from degenerative alterations, it seems reasonable to assume a similar causative mechanism. ${ }^{3031}$ In subjects without a transition point in the resting blood pressure range (normal subjects and the majority of Marfan patients), the load bearing component is elastin. In the six Marfan patients with a transition point, the far stiffer collagen fibres are recruited. A correlation has been shown between a transition point in the pressure-area curve and both susceptibility to permanent dilatation and breaking stress of the thoracic aorta. ${ }^{12}$

\section{Heterogeneous response to $\beta$ blocking agents}

It is generally accepted that $\beta$ blockade protects the Marfan aorta from both dilatation and dissection. Shores et al found a heterogeneous response, with some patients deriving greater

Table 2 Aortic diameters and aortic elasticity in Marfan patients with and without $\beta$ blocking agents

\begin{tabular}{|c|c|c|c|}
\hline & \multicolumn{2}{|l|}{ Marfan patients } & \multirow[b]{2}{*}{ p Value } \\
\hline & $\begin{array}{l}\text { Without } \beta \text { blockade } \\
(n=7)\end{array}$ & $\begin{array}{l}\text { With } \beta \text { blockade } \\
(n=25)\end{array}$ & \\
\hline $\begin{array}{l}\text { Transition point } \\
\text { Diameter of descending thoracic }\end{array}$ & $1(14 \%)$ & $5(20 \%)$ & NS \\
\hline $\begin{array}{l}\text { aorta }(\mathrm{mm}) \\
\text { Distensibility }\left(10^{-3} \mathrm{~mm} \mathrm{Hg}^{-1}\right)\end{array}$ & $\begin{array}{l}24(2) \\
3.8(0.9)\end{array}$ & $\begin{array}{l}24(3) \\
3.2(1.5)\end{array}$ & $\begin{array}{l}\text { NS } \\
\text { NS }\end{array}$ \\
\hline Mean blood pressure $(\mathrm{mm} \mathrm{Hg})$ & $90(6)$ & $86(8)$ & NS \\
\hline
\end{tabular}

Values are mean (SD). 
Table 3 Aortic diameters and aortic elasticity in control subjects and Marfan patients with and without a transition point

\begin{tabular}{|c|c|c|c|c|}
\hline & \multirow[b]{2}{*}{ Controls $(n=10)$} & \multicolumn{2}{|l|}{ Marfan patients } & \multirow[b]{2}{*}{ p Value } \\
\hline & & Without TP $(n=26)$ & With TP $(n=6)$ & \\
\hline $\begin{array}{l}\mathrm{E}_{\mathrm{e}}\left(\mathrm{mm} \mathrm{Hg} / \mathrm{mm}^{2}\right) \\
\text { Diameter descending thoracic }\end{array}$ & $0.46(0.14)$ & $0.49(0.21)$ & $0.36(0.10)$ & NS \\
\hline aorta (mm) & $20(3)$ & $24(2)$ & $24(2)$ & NS \\
\hline Distensibility $\left(10^{-3} \mathrm{~mm} \mathrm{Hg}^{-1}\right)$ & $4.6(0.9)$ & $3.6(1.5)$ & $2.6(0.7)$ & NS \\
\hline Mean blood pressure $(\mathrm{mm} \mathrm{Hg})$ & $86(7)$ & $87(8)$ & $86(5)$ & NS \\
\hline
\end{tabular}

benefit from $\beta$ blockade (that is, retardation of aortic dilatation), whereas in others the course of the disease seemed to be unaffected. ${ }^{6}$ A retrospective historically controlled trial of propranolol or atenolol treatment in 113 patients found similar effects. ${ }^{7}$ The effect of $\beta$ blockade on aortic elasticity in Marfan patients has been described in several studies..$^{910}$ Aortic distensibility is decreased in Marfan disease and the values in our study are comparable with those previously reported. ${ }^{92-34}$ The effect of $\beta$ blockade on the aortic elastic properties has been assessed noninvasively by Groenink et al, comparing normal controls with patients with Marfan syndrome after two weeks of $\beta$ blocker treatment. ${ }^{9}$ They observed a significant decrease in aortic elasticity only in the Marfan patients. ${ }^{9}$ Haouzi et al found a heterogeneous response on aortic root elasticity after a single dose of metoprolol in patients with Marfan syndrome. ${ }^{10}$ Rios et al also found a non-uniform response to long term oral atenolol, although as a group there was a significant increase in distensibility and a trend toward lowering the stiffness index in the Marfan patients. ${ }^{11}$ An important observation described by Yin et al was that in some patients with moderate to severe dilatation of the aortic root, worsening of aortic properties occurred when they were taking $\beta$ blocking agents, raising questions about the beneficial prophylactic effect. ${ }^{35}$

The presence of a transition point in the pressure-area curve may play a crucial role in the heterogeneous response to $\beta$ blockade in Marfan patients. In view of the pressurearea relation of the great arteries, ${ }^{36}$ a decrease in mean blood pressure following $\beta$ blockade could result in increased distensibility when systolic aortic area shifts from the collagen determined (exponential) part of the pressure-area curve to the elastin (linear) determined part (fig 3). When $\beta$ blockade causes a decrease in blood pressure in the linear part of the curve, distensibility is not expected to increase because the area change remains similar. We found that in the majority of patients with Marfan syndrome and in all our controls, the pressure-area curve did not show a transition point. Because the slope of the linear part of the pressurearea curve was similar to the slope of normal subjects, it is reasonable to assume that the transition point in these subjects was at a higher aortic pressure than their resting blood pressure. Only in six patients with Marfan syndrome was a transition point in the pressure-area curve observed, indicating that the load bearing component in the resting blood pressure range involved collagen as well as elastin. In these patients the transition point was shifted to much lower pressures, which could indicate more pronounced degeneration of the medial layer of the aortic wall than in patients without a transition point. This suggests that the presence of a transition point in the pressure-area curve could play a role in the heterogeneous response to $\beta$ blocker treatment in relation to the elastic properties and dilatation rate. The transition point in the pressure-area curve in Marfan patients

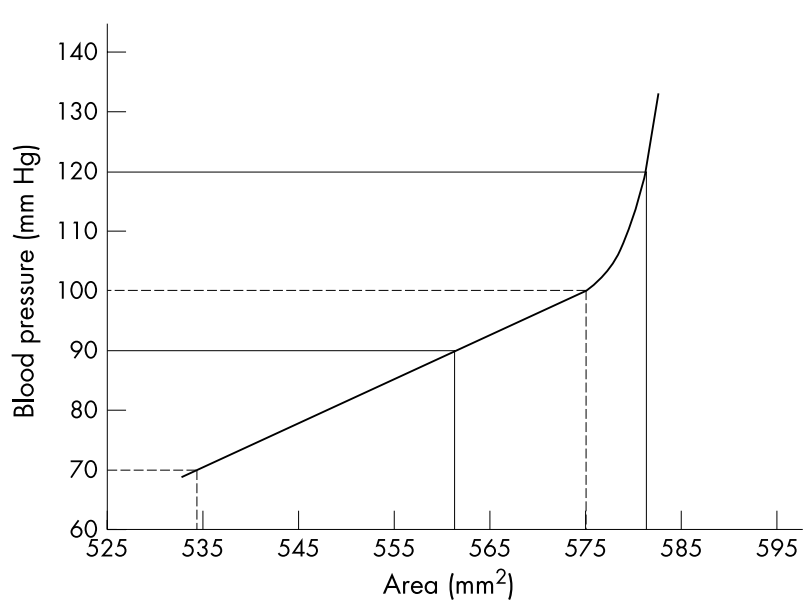

Figure 3 Curve of the aortic pressure-area relation in a patient with Marfan syndrome and the influence of lowering the blood pressure on distensibility. The solid lines indicate the aortic area change at a blood pressure of $120 / 90 \mathrm{~mm} \mathrm{Hg}$. Lowering the blood pressure to $100 / 70$ $\mathrm{mm} \mathrm{Hg}$ (dotted lines) will result in an increase in area change, because the working area is shifted to the linear part of the curve.

may vary as a result of different degrees of aortic media degeneration. Patients with a transition point at lower blood pressures are not expected to benefit from $\beta$ blocker treatment.

\section{Limitations of the study}

The use of $\beta$ blocking agents was not equally distributed between the two groups-there were considerably more patients in the group with $\beta$ blocking agents than in the group without (27 $\vee 7$ patients). The lack of a difference observed between patients with and without $\beta$ blocker treatment may reflect the small sample size.

Furthermore, blood pressure and area should ideally be measured simultaneously and at the same level in the aorta for reliable assessment of pressure-area curves. MRI and Finometer blood pressure recordings were done separately, because it is not yet possible to use the Finometer front end in the strong magnetic field of the magnetic resonance scanner. However, as Marfan patients regularly undergo MRI for monitoring aortic dimensions, physical and emotional stress during the procedure is limited. This was confirmed by similar blood pressure and heart rates during both MRI and Finometer recording, indicating comparable conditions. Although the Finometer blood pressure recordings were not at the site of the area measurement, the filter developed on the basis of unpublished data corresponds well with other pulse wave filters from brachial artery to aorta described in published reports. ${ }^{18-20}$ 


\section{Future developments}

Adaptation of the Finometer device to ensure reliable function in the strong magnetic field of the magnetic resonance scanner-combined with new developments in MRI as "real time," or techniques with a smaller temporal resolution-will further improve the non-invasive assessment of pressure-area curves in the near future. Until the Finometer is adapted for use in the magnetic resonance scanner, the method can be validated by means of transoesophageal echocardiography combined with noninvasive or invasive aortic pressure measurement. Further prospective randomised research on the effect of $\beta$ blocker treatment on the aortic pressure-area relation in Marfan patients is required.

\section{Conclusions}

Our new non-invasive method to derive aortic pressure-area curves showed that the great majority of patients with Marfan syndrome on $\beta$ blocking agents had pressure-area curves similar to control subjects with comparable blood pressures. The variable position of the transition point in the aortic pressure-area curve in individual patients could play a crucial role in the heterogeneous response to $\beta$ blockade in Marfan patients. We hypothesise that patients with a transition at low blood pressures will not benefit from $\beta$ blocking agents.

\section{Authors' affiliations}

G J Nollen, M Groenink, A Osnabrugge, B J M Mulder, Department of Cardiology, Academic Medical Centre, Amsterdam, Netherlands

B E Westerhof, Department of TNO Biomedical Instrumentation, Academic Medical Centre, Amsterdam

E E van der Wall, Department of Cardiology, Leiden University Medical Centre, Leiden, Netherlands

\section{REFERENCES}

1 Dietz HC, Cutting GR, Pyeritz RE, et al. Marfan syndrome caused by a recurrent de novo missense mutation in the fibrillin gene. Nature 1991;352:337-9

2 Murdoch JL, Walker BA, Halpern BL, et al. Life expectancy and causes of death in the Marfan syndrome. N Engl J Med 1972;286:804-8.

3 Silverman DI, Burton KJ, Gray JJ, et al. Life expectancy in the Marfan syndrome. Am J Cardiol 1995;75:157-60.

4 Sütch G, Jenni R, von Segesser L, et al. Predictability of aortic dissection as a function of aortic diameter. Eur Heart $J$ 1991;12:1247-56.

5 Groenink M, Lohuis TAJ, Tijssen JGP, et al. Survival and complication-free survival in Marfan's syndrome: implications of current guidelines. Heart 1999;82:499-504.

6 Shores J, Berger KR, Murphy EA, et al. Progression of aortic dilatation and the benefit of long-term beta-adrenergic blockade in Marfan's syndrome. N Engl J Med 1994:330:1335-41.

7 Salim MA, Alpert BS, Ward JC, et al. Effect of beta-adrenergic blockade on aortic root rate of dilation in the Marfan syndrome. Am J Cardiol 1994;74:629-33.

8 Marsalese DL, Moodie DS, Vacante M, et al. Marfan's syndrome: natural history and long-term follow-up of cardiovascular involvement. J Am Coll Cardiol 1989; 14:422-8.

9 Groenink M, de Roos A, Mulder BJM, et al. Changes in aortic distensibility and pulse wave velocity assessed with magnetic resonance imaging following beta-blocker therapy in the Marfan syndrome. Am J Cardiol 1998;82:203-8.
10 Haouzi A, Berglund H, Pelikan PC, et al. Heterogeneous aortic response to acute beta-adrenergic blockade in Marfan syndrome. Am Heart J 1997; 133:60-3.

11 Rios AS, Silber EN, Bavishi N, et al. Effect of long-term beta blockade on aortic root compliance in patients with Marfan syndrome. Am Heart J 1999; 137:1057-61.

12 Groenink M, Langerak SE, Vanbavel E, et al. The influence of aging and aortic stiffness on the resistance to permanent dilation and breaking stress of the thoracic descending aorta. Cardiovasc Res 1999;43:471-80.

13 Bader H. Dependence of wall stress in the human thoracic aorta on age and pressure. Circulation Res 1967;20:354-61.

14 Imholz BP, Wieling W, van Montfrans GA, et al. Fifteen years experience with finger arterial pressure monitoring: assessment of the technology. Cardiovasc Res 1998; 38:605-16.

15 DePaepe A, Devereux RB, Dietz HC, et al. Revised diagnostic criteria for the Marfan syndrome. Am J Med Genet 1996;62:417-26.

16 Bos WJ, van Goudoever J, van Montfrans GA, et al. Reconstruction of brachial artery pressure from noninvasive finger pressure measurements. Circulation 1996:94:1870-7.

17 Guelen I, Westerhof BE, Van Der Sar GL, et al. Finometer, finger pressure measurements with the possibility to reconstruct brachial pressure. Blood Press Monit 2003;8:27-30.

18 Lasance HAJ, Wesseling KH, Ascoop CA. Peripheral pulse contour analysis in determining stroke volume. In: Progress report 5, Institute of Medical Physics. Utrecht: Institute of Medical Physics, 1976:59-62.

19 Karamanoglu M, O'Rourke MF, Avolio AP, et al. An analysis of the relationship between central aortic and peripheral upper limb pressure waves in man. Eur Heart J 1993;14:160-7.

20 Chen $\mathrm{CH}$, Nevo E, Fetics B, et al. Estimation of central aortic pressure waveform by mathematical transformation of radial tonometry pressure. Circulation 1997;95:1827-36.

21 Sugawara M, Niki K, Furuhata $\mathrm{H}$, et al. Relationship between the pressure and diameter of the carotid artery in humans. Heart Vessels 2000;15:49-51.

22 van Popele NM, Bos WJW, de Beer NAM, et al. Arterial stiffness as underlying mechanism of disagreement between an oscillometric blood pressure monitor and a sphygmomanometer. Hypertension 2000;36:484-8.

23 Hasan MA, Thomas TA, Prys-Roberts C. Comparison of Automated oscillometric arterial pressure measurement with conventional auscultatory measurement in the labour ward. Br J Anaesth 1993;70:141-4.

24 Bogren HG, Mohiaddin RH, Klipstein RK, et al. The function of the aorta in ischemic heart disease: a magnetic resonance and angiographic study of aortic compliance and blood flow patterns. Am Heart J 1989;1 18:234-47

25 Heintz B, Gillessen T, Walkenhorst F, et al. Evaluation of segmental elastic properties of the aorta in normotensive and medically treated hypertensive patients by intravascular ultrasound. J Hypertens 1993;11:1253-8.

26 Stefanadis C, Stratos C, Vlachopoulos C, et al. Pressure-diameter relation of the human aorta. A new method of determination by the application of a special ultrasonic dimension catheter. Circulation 1995;92:2210-19.

27 Honda T, Yano K, Matsuoka H, et al. Evaluation of aortic distensibility in patients with essential hypertension by using cine magnetic resonance imaging. Angiology 1994;45:207-12.

28 Mohiaddin RH, Firmin DN, Longmore DB. Age-related changes of human aortic flow wave velocity measured noninvasively by magnetic resonance imaging. J Appl Physiol 1993;74:492-7.

29 Roy CS. Elastic properties of the arterial wall. J Physiol (Lond) 1880;3:125-59.

30 Carlson RS, Lillehei CW, Edwards JE. Cystic media necrosis of ascending aorta in relation to age and hypertension. Am J Cardiol 1970;25:411.

31 Schlatmann TJM, Becker AE. Histologic changes in the normal aging aorta: implications for dissecting aortic aneurysm. Am J Cardiol 1977;39:13-20.

32 Adams JN, Brooks M, Redpath TW, et al. Aortic distensibility measured by magnetic resonance imaging in patients with Marfan's syndrome. $\mathrm{Br}$ Heart $J$ 1995; 73:265-9.

33 Hirata K, Triposkiadis F, Sparks E, et al. The Marfan syndrome: abnormal elastic properties. J Am Coll Cardiol 1991; 18:57-63.

34 Groenink M, de Roos A, Mulder BJM, et al. Biophysical properties of the normal-sized aorta in patients with Marfan syndrome: evaluation with MR flow mapping. Radiology 2001;219:535-40.

35 Yin F, Brin K, Ting C, et al. Arterial hemodynamic indexes in Marfan's syndrome. Circulation 1989;79:854-62.

36 Greenfield JC, Patel DJ. Relation between pressure and diameter in the ascending aorta of man. Circ Res 1962;10:778-81. 